\title{
Chemical Weed Control in Chilli + Onion Intercropping System
}

\author{
Vilas D. Gasti* and Snehasish Chakravorty \\ Department of Horticulture and Post-harvest Technology Palli Siksha Bhavana (Institute of \\ Agriculture) Sriniketan, Visva-Bharati (West Bengal) - 731236, India
}

*Corresponding author

\begin{abstract}
A B S T R A C T
Keywords

Chilli + onion intercropping,

Weed control treatments and $\mathrm{B}$ : C Ratio

Article Info

Accepted:

26 December 2018

Available Online:

10 January 2019

An investigation entitled "Chemical weed control in chilli + onion intercropping system" was carried out at the Vegetable Division in Kittur Rani Channamma College of Horticulture, Arabhavi, University of Horticultural Sciences, Bagalkot (Karnataka) during Kharif season of 2014 and 2015 on well drained red loamy soil to find out the suitable approach (herbicides+cultural practices) for weed management and for improving the productivity of chilli+onion intercropping system. Observations on weed control treatments showed marked difference among weed, growth and yield parameters in chilli and onion. Among the different chemical treatments, pre-emergent application of alachlor @ $1.5 \mathrm{~kg}$ ai/ha $+2 \mathrm{HW}$ at 45 and $60 \mathrm{DAT}\left(\mathrm{T}_{4}\right)$ recorded the lowest pooled dry matter of weeds (1.97 g), lowest population of monocot (3.68) and dicot weeds (3.11) and thus exhibited the highest weed control efficiency (93.04\%). Unweeded check recorded highest weed population (13.16) and weed dry weight $(7.47 \mathrm{~g})$. Significantly higher yield per ha of green chilli(253.41 q) and onion (221.26 q), net returns (Rs.2,14,413) and B:C ratio (3.4) was recorded in $\mathrm{T}_{4}$ followed by pendimethalin @ $1.5 \mathrm{~kg}$ ai $/ \mathrm{ha}+2 \mathrm{HW}$ at 45 and $60 \mathrm{DAT}$.
\end{abstract}

\section{Introduction}

Weeds are unwanted and undesirable plants that interfere with utilization of land and water resources and thus adversely affect crop production and human welfare. They are often prolific and persistent, interfere with agricultural operations, increase labour cost and reduce the yield (up to $45 \%$ ) and quality. The weed flora noticed in the experimental site consisted of grasses, sedges and broad leaved weed category. The important grassy weeds observed are Cynodon dactylon,
Dinebra retroflexa, Eleusine indica, Cyperus rotundus and Setaria italica. C. rotundus was more dominated than other weeds in entire experimental area. In Karnataka, chilli and onion are the most extensively grown commercial vegetable crops under intercropping systems. Chilli+onion intercropping is of the most assured intercropping system and mitigate the weeds to some extent and increases the yield and is found suitable to northern dry zones of Karnataka. This system is widely adopted in Northern Karnataka (Anonymous, 2017). 
In general, weeds are considered to be one of the major production constraints in chilli and onion. Timely weeding reduces the crop weed competition and leads to higher crop yields. Hand weeding, though an efficient method it is laborious, costly, time consuming and unsuitable for large farms. The cost involved in hand weeding and unavailability of labours for manual weeding has necessitated the use of chemicals for weed control in developing countries like India. Information regarding weed management through herbicides in intercropping is limited and very little work has been done on weed management in chilli+ onion intercropping system in particular. In view of acute early results to mitigate weeds through chemical weed management strategy for chilli + onion intercropping system, the present investigation was carried out.

Chilli and onion is the most important cash crops of India, playing a key role in economic and social affairs. In intercropping system, fewer weeds are expected than in sole crop because of their better suppression. Some research findings related to weed management reported that weed suppression as a result of intercropping is to the extent of 50 to 75 per cent (Biradar, 1999). However management of weeds in intercropping or mixed cropping system is complicated due to spatial and temporal differences in the arrangement of crops. Shallow root system of onion prevents mechanical weeding.

Smothering effect of intercrop in onion is absent due to slow initial growth. Hence, chemical weed control is also difficult due to simultaneous sowing of two or more crops but selective herbicides play a key role in suppression of weeds in intercropping due to simultaneous suppression of weeds by smothering effect (Muthusankaranarayanan et al., 1997). Large stretches of chilli + onion intercropping system in northern dry zone of Karnataka is indicative of its high productiveness and certainly in obtaining the yields. Mechanical weeding is difficult as the onion rows are closely spaced are often planted in both the directions as practiced by farmers of this region.

\section{Materials and Methods}

The study was carried out at the Vegetable Division in Kittur Rani Channamma College of Horticulture, Arabhavi, University of Horticultural Sciences, Bagalkot (Karnataka) during Kharif season of 2014 and 2015 on a well-drained red loamy soil to find out the effectiveness of chemicals in weed management in intercropping of chilli (Var 'Byadagi')+ onion (Var 'N-53').

The field experiment was laid out in a Randomized Complete Block Design with two replications consisting of fourteen treatments including unweeded check $\left(\mathrm{T}_{1}\right.$ Alachlor (PE)-1.5 kg ai/ha $\mathrm{T}_{2}$ - Alachlor (PE)$1.0 \mathrm{~kg}$ ai $/ \mathrm{ha} \mathrm{T}_{3}$. Alachlor (PE)-1.5 kg ai/ha + $1 \mathrm{HW}$ at $45 \mathrm{DAT} \mathrm{T}_{4}$ - Alachlor (PE)-1.5 kg ai/ha $+2 \mathrm{HW}$ at 45 and $60 \mathrm{DAT} \mathrm{T}_{5}$ - Alachlor (PE)-1.0 kg ai/ha + $1 \mathrm{HW}$ at $45 \mathrm{DAT} \mathrm{T}_{6}$ Alachlor (PE)-1.0 kg ai/ha $+2 \mathrm{HW}$ at 45 and 60 DAT $\mathrm{T}_{7}$ - Pendimethalin (PE) $-1.5 \mathrm{~kg}$ ai $/ \mathrm{ha}$ $\mathrm{T}_{8}$ - Pendimethalin (PE)-1.0 kg ai/ha $\mathrm{T}_{9}$ Pendimethalin (PE)-1.5 kg ai/ha+ $1 \mathrm{HW}$ at 45 DAT $\mathrm{T}_{10}$ - Pendimethalin (PE) $1.5 \mathrm{~kg}$ ai $/ \mathrm{ha}+$ $2 \mathrm{HW}$ at 45 and 60 DAT T 11 . Pendimethalin (PE)-1.0 kg ai/ha + $1 \mathrm{HW}$ at $45 \mathrm{DAT} \mathrm{T}_{12}$ Pendimethalin (PE)-1.0 kg ai/ha $+2 \mathrm{HW}$ at 45 and 60 DAT $T_{13}$. Weed free check $T_{14}$ Unweeded check). RDF for chilli is 150:100:125 kg of $\mathrm{N}: \mathrm{P}_{2} \mathrm{O}_{5}: \mathrm{K}_{2} \mathrm{O}$ with 25 tonnes of FYM (As per package of practices UHS, Bagalkot Karnataka).

The gross size and net size of the plots were $16.20 \mathrm{~m}^{2}$ and $11.80 \mathrm{~m}^{2}$ respectively. Four to five weeks old chilli seedlings are transplanted into main field with a spacing of $75 \mathrm{~cm} \times 45 \mathrm{~cm}$ (As per package of practices - 
UHS, Bagalkot) and in between the chilli plants, 2 rows of onion was planted. Basal dose of fertilizer $(50 \% \mathrm{~N}$ and full dose of $\mathrm{P}$ and $\mathrm{K}$ ) was applied to each treatment at the time of field preparation. Remaining dose of $\mathrm{N}(50 \%)$ was applied at 3 split doses viz., after 30, 60 and 90 days of transplanting. During the course of investigation, observations regarding weed population, growth and yield parameters at 30,60 and 90 DAT and at the time of harvest were recorded from the randomly selected and tagged plants.

The weed index was calculated by the formula given by Gill and Vijayakumar (1969). Besides fixed cost of cultivation, variable costs on spray, manual weeding and cost of herbicide in each treatment was worked out to obtain total cost of production. The net income was obtained after deducting cost of production from value of produces. The mean data was subjected to the statistical analysis using ANOVA and mean separation (LSD) procedures (Gomez and Gomez, 1984).

\section{Results and Discussion}

The results of the study showed that among different chemical treatments, the effect of weed control on monocot weeds (3.68), dicot weeds (3.11) and weed population at harvest (4.82) was found to be significantly less in treatment with pre-emergent application of alachlor- $1.5 \mathrm{~kg}$ ai/ha $+2 \mathrm{HW}$ at 45 and 60 $\operatorname{DAT}\left(\mathrm{T}_{4}\right)$ over unweeded check ie, $\mathrm{T}_{14}$. This was found to be on par with $T_{10}$ (Pendimethalin (PE)-1.5 kg ai/ha $+2 \mathrm{HW}$ at 45 and 60 DAT) (3.95, 3.26 and 5.12, respectively) (Table 1 ).

The treatment $\mathrm{T}_{4}$ recorded lowest dry weight of weeds at harvest $(1.97 \mathrm{~g})$, lowest weed index in chilli and onion (11.80 and 6.05, respectively) and thus highest weed control efficiency (93.04\%) (Table 2). The lower dry weight of weeds in these treatments might be attributed to the less number of weeds. Thus the higher weed control efficiency could be accounted to the lower weed dry weight. These results are of agreement with Ningappa (2013), Shil and Adhikary (2014) and Chaudhari et al., (2017).

Treatment $\mathrm{T}_{4}$ was found significant for highest plant height $(104.88 \mathrm{~cm})$, number of branches/ plant (24.47), dry weight of plant $(106.39 \mathrm{~g})$ and number of fruits/plant at harvest (119.34) in chilli (Table 3). The mean analyses showed that highest fruit wt. /plant $(1.08 \mathrm{~kg})$ was recorded in $\mathrm{T}_{4}$ followed by $\mathrm{T}_{10}$ $(1.04 \mathrm{~kg})$ and minimum was recorded from the unweeded check, $\mathrm{T}_{14}(0.41 \mathrm{~kg})$. Reduced yield from the unweeded plot may be attributed to increased competition for light, soil moisture and nutrients. Fruit yield per ha (253.41 q) of chilli was found highest in $\mathrm{T}_{4}$ while lowest was found in $\mathrm{T}_{14}(101.45 \mathrm{q})$ (Table 4). Similar findings are reported by Rajakumara (2009), Kalasare et al., (2016) and Ningappa (2013).

Yield and yield components of onion varied significantly among various weed control treatments. Treatment $\mathrm{T}_{4}$ was found significant for highest plant height $(59.60 \mathrm{~cm})$ and number of leaves/plant at harvest (15.29) in onion (Table 5). Weed free check recorded significantly highest dry weight of plant $(8.00$ $\mathrm{g})$, yield per plant $(84.80 \mathrm{~g})$ and yield per ha (235.54 q). Among the chemical treatments, $\mathrm{T}_{4}$ recorded highest dry weight of plant (7.57 $\mathrm{g}$ ), yield per plant (79.66 g) and yield per ha (221.26 q) (Table 6).

The increase in plant dry matter and yield per plant in these treatments could be attributed to lower weed count and higher weed control efficiency which ultimately resulted in better crop growth leading to higher productivity. The results are in agreement with Dharmatti et al., (2008), Kalasare (2016) and Urraiya and Jha (2018). 
Table.1 Effect of weed control treatments on weed parameters in chilli + onion intercropping

\begin{tabular}{|c|c|c|c|c|c|c|c|c|c|c|}
\hline \multirow[t]{2}{*}{ Treaments } & \multirow{2}{*}{ Treatment details } & \multicolumn{3}{|c|}{ Monocot weeds at harvest } & \multicolumn{3}{|c|}{ Dicot weeds at harvest } & \multicolumn{3}{|c|}{ Weed population at harvest } \\
\hline & & $\begin{array}{l}\text { I } \\
\text { year }\end{array}$ & $\begin{array}{l}\text { II } \\
\text { year }\end{array}$ & pooled & $\begin{array}{l}\text { I } \\
\text { year }\end{array}$ & $\begin{array}{l}\text { II } \\
\text { year }\end{array}$ & Pooled & $\begin{array}{l}\text { I } \\
\text { year }\end{array}$ & $\begin{array}{l}\text { II } \\
\text { year }\end{array}$ & Pooled \\
\hline $\mathbf{T}_{1}$ & Alachlor (PE)-1.5 kg ai/ha & $\begin{array}{l}54.15 \\
(7.36)\end{array}$ & $\begin{array}{l}55.87 \\
(7.47) \\
\end{array}$ & $\begin{array}{l}55.01 \\
(7.42)\end{array}$ & $\begin{array}{l}28.35 \\
(5.32)\end{array}$ & $\begin{array}{l}29.93 \\
(5.47)\end{array}$ & $\begin{array}{l}29.14 \\
(5.40)\end{array}$ & $\begin{array}{l}82.50 \\
(9.08)\end{array}$ & $\begin{array}{l}85.80 \\
(9.26)\end{array}$ & $\begin{array}{l}84.15 \\
(9.17)\end{array}$ \\
\hline $\mathbf{T}_{\mathbf{2}}$ & Alachlor (PE)-1.0 kg ai/ha & $\begin{array}{l}63.25 \\
(7.95) \\
\end{array}$ & $\begin{array}{l}64.95 \\
(8.06) \\
\end{array}$ & $\begin{array}{l}64.10 \\
(8.00)\end{array}$ & $\begin{array}{l}37.15 \\
(6.09)\end{array}$ & $\begin{array}{l}38.72 \\
(6.22)\end{array}$ & $\begin{array}{l}37.93 \\
(6.16)\end{array}$ & $\begin{array}{l}100.40 \\
(10.02)\end{array}$ & $\begin{array}{l}103.67 \\
(10.18)\end{array}$ & $\begin{array}{l}102.03 \\
(10.10)\end{array}$ \\
\hline $\mathbf{T}_{3}$ & Alachlor $(\mathrm{PE})-1.5 \mathrm{~kg}$ ai $/ \mathrm{ha}+1 \mathrm{HW}$ at $45 \mathrm{DAT}$ & $\begin{array}{l}37.45 \\
(6.12)\end{array}$ & $\begin{array}{l}39.16 \\
(6.26)\end{array}$ & $\begin{array}{l}38.30 \\
(6.19)\end{array}$ & $\begin{array}{l}17.41 \\
(4.17)\end{array}$ & $\begin{array}{l}18.99 \\
(4.36)\end{array}$ & $\begin{array}{l}18.20 \\
(4.26)\end{array}$ & $\begin{array}{l}54.86 \\
(7.41)\end{array}$ & $\begin{array}{l}58.15 \\
(7.62)\end{array}$ & $\begin{array}{l}56.50 \\
(7.52)\end{array}$ \\
\hline $\mathbf{T}_{4}$ & Alachlor $(\mathrm{PE})-1.5 \mathrm{~kg}$ ai $/ \mathrm{ha}+2 \mathrm{HW}$ at 45 and $60 \mathrm{DAT}$ & $\begin{array}{l}12.68 \\
(3.56)\end{array}$ & $\begin{array}{l}14.35 \\
(3.79)\end{array}$ & $\begin{array}{l}13.52 \\
(3.68)\end{array}$ & $\begin{array}{l}8.89 \\
(2.98)\end{array}$ & $\begin{array}{l}10.51 \\
(3.24)\end{array}$ & $\begin{array}{l}9.70 \\
(3.11)\end{array}$ & $\begin{array}{l}21.57 \\
(4.64)\end{array}$ & $\begin{array}{l}24.86 \\
(4.99)\end{array}$ & $\begin{array}{l}23.21 \\
(4.82)\end{array}$ \\
\hline $\mathbf{T}_{5}$ & Alachlor $(\mathrm{PE})-1.0 \mathrm{~kg}$ ai $/ \mathrm{ha}+1 \mathrm{HW}$ at $45 \mathrm{DAT}$ & $\begin{array}{l}42.15 \\
(6.49)\end{array}$ & $\begin{array}{l}43.86 \\
(6.62)\end{array}$ & $\begin{array}{l}43.00 \\
(6.56)\end{array}$ & $\begin{array}{l}19.38 \\
(4.40)\end{array}$ & $\begin{array}{l}21.00 \\
(4.58)\end{array}$ & $\begin{array}{l}20.19 \\
(4.49)\end{array}$ & $\begin{array}{l}61.53 \\
(7.84)\end{array}$ & $\begin{array}{l}64.86 \\
(8.05)\end{array}$ & $\begin{array}{l}63.19 \\
(7.95)\end{array}$ \\
\hline $\mathbf{T}_{6}$ & Alachlor $(\mathrm{PE})-1.0 \mathrm{~kg}$ ai $/ \mathrm{ha}+2 \mathrm{HW}$ at 45 and $60 \mathrm{DAT}$ & $\begin{array}{l}28.88 \\
(5.37)\end{array}$ & $\begin{array}{l}30.56 \\
(5.53)\end{array}$ & $\begin{array}{l}29.72 \\
(5.45)\end{array}$ & $\begin{array}{l}10.80 \\
(3.28)\end{array}$ & $\begin{array}{l}12.37 \\
(3.52)\end{array}$ & $\begin{array}{l}11.59 \\
(3.40)\end{array}$ & $\begin{array}{l}39.68 \\
(6.30)\end{array}$ & $\begin{array}{l}42.93 \\
(6.55)\end{array}$ & $\begin{array}{l}41.31 \\
(6.43)\end{array}$ \\
\hline $\mathbf{T}_{7}$ & Pendimethalin (PE)-1.5 kg ai/ha & $\begin{array}{l}60.95 \\
(7.81) \\
\end{array}$ & $\begin{array}{l}62.68 \\
(7.92) \\
\end{array}$ & $\begin{array}{l}61.82 \\
(7.86) \\
\end{array}$ & $\begin{array}{l}33.15 \\
(5.76) \\
\end{array}$ & $\begin{array}{l}34.73 \\
(5.89) \\
\end{array}$ & $\begin{array}{l}33.94 \\
(5.83) \\
\end{array}$ & $\begin{array}{l}94.10 \\
(9.70)\end{array}$ & $\begin{array}{l}97.41 \\
(9.87)\end{array}$ & $\begin{array}{l}95.75 \\
(9.78)\end{array}$ \\
\hline $\mathbf{T}_{\mathbf{8}}$ & Pendimethalin (PE)-1.0 kg ai/ha & $\begin{array}{l}67.15 \\
(8.19) \\
\end{array}$ & $\begin{array}{l}68.88 \\
(8.30) \\
\end{array}$ & $\begin{array}{l}68.01 \\
(8.25)\end{array}$ & $\begin{array}{l}40.18 \\
(6.34) \\
\end{array}$ & $\begin{array}{l}41.81 \\
(6.47) \\
\end{array}$ & $\begin{array}{l}40.99 \\
(6.40)\end{array}$ & $\begin{array}{l}107.33 \\
(10.36)\end{array}$ & $\begin{array}{l}110.68 \\
(10.52)\end{array}$ & $\begin{array}{l}109.01 \\
(10.44)\end{array}$ \\
\hline $\mathbf{T}_{9}$ & Pendimethalin (PE)-1.5 kg ai/ha+ $1 \mathrm{HW}$ at $45 \mathrm{DAT}$ & $\begin{array}{l}45.38 \\
(6.74)\end{array}$ & $\begin{array}{l}47.10 \\
(6.86)\end{array}$ & $\begin{array}{l}46.24 \\
(6.80)\end{array}$ & $\begin{array}{l}20.90 \\
(4.57)\end{array}$ & $\begin{array}{l}22.51 \\
(4.74)\end{array}$ & $\begin{array}{l}21.70 \\
(4.66)\end{array}$ & $\begin{array}{l}66.28 \\
(8.14)\end{array}$ & $\begin{array}{l}69.61 \\
(8.34)\end{array}$ & $\begin{array}{l}67.94 \\
(8.24)\end{array}$ \\
\hline $\mathbf{T}_{10}$ & $\begin{array}{l}\text { Pendimethalin }(\mathrm{PE})-1.5 \mathrm{~kg} \text { ai/ha }+2 \mathrm{HW} \text { at } 45 \text { and } 60 \\
\text { DAT }\end{array}$ & $\begin{array}{l}14.70 \\
(3.83)\end{array}$ & $\begin{array}{l}16.44 \\
(4.05)\end{array}$ & $\begin{array}{l}15.57 \\
(3.95)\end{array}$ & $\begin{array}{l}9.85 \\
(3.14)\end{array}$ & $\begin{array}{l}11.45 \\
(3.38)\end{array}$ & $\begin{array}{l}10.65 \\
(3.26)\end{array}$ & $\begin{array}{l}24.55 \\
(4.95)\end{array}$ & $\begin{array}{l}27.89 \\
(5.28)\end{array}$ & $\begin{array}{l}26.22 \\
(5.12)\end{array}$ \\
\hline $\mathbf{T}_{11}$ & Pendimethalin (PE)-1.0 kg ai/ha + $1 \mathrm{HW}$ at $45 \mathrm{DAT}$ & $\begin{array}{l}53.58 \\
(7.32)\end{array}$ & $\begin{array}{l}55.28 \\
(7.44)\end{array}$ & $\begin{array}{l}54.43 \\
(7.38)\end{array}$ & $\begin{array}{l}23.98 \\
(4.90)\end{array}$ & $\begin{array}{l}25.59 \\
(5.06)\end{array}$ & $\begin{array}{l}24.79 \\
(4.98)\end{array}$ & $\begin{array}{l}77.56 \\
(8.81)\end{array}$ & $\begin{array}{l}80.87 \\
(8.99)\end{array}$ & $\begin{array}{l}79.22 \\
(8.90)\end{array}$ \\
\hline $\mathbf{T}_{12}$ & $\begin{array}{l}\text { Pendimethalin (PE)-1.0 kg ai/ha }+2 \mathrm{HW} \text { at } 45 \text { and } 60 \\
\text { DAT }\end{array}$ & $\begin{array}{l}31.45 \\
(5.61)\end{array}$ & $\begin{array}{l}33.40 \\
(5.78)\end{array}$ & $\begin{array}{l}32.43 \\
(5.69)\end{array}$ & $\begin{array}{l}12.46 \\
(3.53)\end{array}$ & $\begin{array}{l}14.04 \\
(3.74)\end{array}$ & $\begin{array}{l}13.25 \\
(3.64)\end{array}$ & $\begin{array}{l}43.91 \\
(6.62)\end{array}$ & $\begin{array}{l}47.44 \\
(6.89)\end{array}$ & $\begin{array}{l}45.67 \\
(6.76)\end{array}$ \\
\hline $\mathbf{T}_{13}$ & Weed free check & $\begin{array}{l}0.00 \\
(0.71)\end{array}$ & $\begin{array}{l}0.00 \\
(0.71)\end{array}$ & $\begin{array}{l}0.00 \\
(0.71)\end{array}$ & $\begin{array}{l}0.00 \\
(0.71) \\
\end{array}$ & $\begin{array}{l}0.00 \\
(0.71) \\
\end{array}$ & $\begin{array}{l}0.00 \\
(0.71)\end{array}$ & $\begin{array}{l}0.00 \\
(0.71)\end{array}$ & $\begin{array}{l}0.00 \\
(0.71)\end{array}$ & $\begin{array}{l}0.00 \\
(0.71)\end{array}$ \\
\hline $\mathbf{T}_{14}$ & Unweeded check & $\begin{array}{l}106.18 \\
(10.30)\end{array}$ & $\begin{array}{l}107.89 \\
(10.38)\end{array}$ & $\begin{array}{l}107.03 \\
(10.34)\end{array}$ & $\begin{array}{l}65.41 \\
(8.09) \\
\end{array}$ & $\begin{array}{l}67.01 \\
(8.19) \\
\end{array}$ & $\begin{array}{l}66.21 \\
(8.14)\end{array}$ & $\begin{array}{l}171.59 \\
(13.10)\end{array}$ & $\begin{array}{l}174.89 \\
(13.22)\end{array}$ & $\begin{array}{l}173.24 \\
(13.16)\end{array}$ \\
\hline \multicolumn{2}{|l|}{ Mean } & $\begin{array}{l}44.14 \\
(6.24)\end{array}$ & $\begin{array}{l}\mathbf{4 5 . 7 4} \\
(6.37)\end{array}$ & $\begin{array}{l}44.94 \\
(6.30)\end{array}$ & $\begin{array}{l}23.42 \\
(4.52)\end{array}$ & $\begin{array}{l}23.42 \\
(4.52)\end{array}$ & $\begin{array}{l}24.16 \\
(4.60)\end{array}$ & $\begin{array}{l}67.56 \\
(7.64)\end{array}$ & $\begin{array}{l}\mathbf{7 0 . 6 8} \\
(7.88)\end{array}$ & $\begin{array}{l}69.12 \\
(7.77)\end{array}$ \\
\hline \multicolumn{2}{|l|}{ S. Em士 } & 0.08 & 0.08 & 0.08 & 0.04 & 0.04 & 0.04 & 0.07 & 0.12 & 0.09 \\
\hline \multicolumn{2}{|l|}{ C.D. @ 5\% } & 0.24 & 0.25 & 0.24 & 0.16 & 0.16 & 0.16 & 0.28 & 0.32 & 0.28 \\
\hline
\end{tabular}


Table.2 Effect of weed control treatments on weed parameters in chilli + onion intercropping

\begin{tabular}{|c|c|c|c|c|c|c|c|c|c|c|c|c|c|}
\hline \multirow[t]{2}{*}{ Treatments } & \multirow[t]{2}{*}{ Treatment details } & \multicolumn{3}{|c|}{$\begin{array}{l}\text { Dry weight of weeds } \\
\text { at harvest (g) }\end{array}$} & \multicolumn{3}{|c|}{$\begin{array}{c}\text { Weed control } \\
\text { efficiency at harvest }\end{array}$} & \multicolumn{3}{|c|}{ Weed index in chilli } & \multicolumn{3}{|c|}{ Weed index in onion } \\
\hline & & $\begin{array}{c}\text { I } \\
\text { year }\end{array}$ & $\begin{array}{c}\text { II } \\
\text { year }\end{array}$ & Pooled & $\begin{array}{c}\text { I } \\
\text { year }\end{array}$ & $\begin{array}{c}\text { II } \\
\text { year }\end{array}$ & Pooled & $\begin{array}{c}\text { I } \\
\text { year }\end{array}$ & $\begin{array}{c}\text { II } \\
\text { year }\end{array}$ & Pooled & $\begin{array}{c}\text { I } \\
\text { year }\end{array}$ & $\begin{array}{c}\text { II } \\
\text { year }\end{array}$ & Pooled \\
\hline $\mathbf{T}_{1}$ & Alachlor (PE)-1.5 kg ai/ha & $\begin{array}{l}31.60 \\
(5.62)\end{array}$ & $\begin{array}{l}32.58 \\
(5.71)\end{array}$ & $\begin{array}{l}32.09 \\
(5.66)\end{array}$ & 42.75 & 42.00 & 42.37 & 19.15 & 19.59 & 19.38 & 16.25 & 17.55 & 16.92 \\
\hline $\mathbf{T}_{2}$ & Alachlor (PE)-1.0 kg ai/ha & $\begin{array}{l}34.19 \\
(5.85)\end{array}$ & $\begin{array}{l}35.19 \\
(5.93)\end{array}$ & $\begin{array}{l}34.69 \\
(5.89)\end{array}$ & 38.15 & 37.43 & 37.79 & 21.30 & 21.73 & 21.52 & 16.82 & 18.34 & 17.60 \\
\hline $\mathbf{T}_{3}$ & Alachlor (PE)-1.5 kg ai $/ \mathrm{ha}+1 \mathrm{HW}$ at $45 \mathrm{DAT}$ & $\begin{array}{l}22.67 \\
(4.76)\end{array}$ & $\begin{array}{l}23.67 \\
(4.86)\end{array}$ & $\begin{array}{l}23.17 \\
(4.81)\end{array}$ & 58.92 & 57.86 & 58.39 & 15.31 & 15.78 & 15.55 & 13.09 & 14.48 & 13.81 \\
\hline $\mathbf{T}_{5}$ & Alachlor (PE)-1.0 kg ai $/ \mathrm{ha}+1 \mathrm{HW}$ at $45 \mathrm{DAT}$ & $\begin{array}{l}24.34 \\
(4.93)\end{array}$ & $\begin{array}{l}25.35 \\
(5.03)\end{array}$ & $\begin{array}{l}24.84 \\
(4.98)\end{array}$ & 55.88 & 54.86 & 55.36 & 15.75 & 16.25 & 16.01 & 15.17 & 16.35 & 15.78 \\
\hline $\mathbf{T}_{6}$ & Alachlor (PE)-1.0 kg ai/ha $+2 \mathrm{HW}$ at 45 and $60 \mathrm{DAT}$ & $\begin{array}{l}10.71 \\
(3.27)\end{array}$ & $\begin{array}{l}11.70 \\
(3.42)\end{array}$ & $\begin{array}{l}11.20 \\
(3.34)\end{array}$ & 80.67 & 79.23 & 79.94 & 13.28 & 13.82 & 13.56 & 7.79 & 9.40 & 8.62 \\
\hline $\mathbf{T}_{7}$ & Pendimethalin (PE)-1.5 kg ai/ha & $\begin{array}{l}33.43 \\
(5.78)\end{array}$ & $\begin{array}{l}34.45 \\
(5.87)\end{array}$ & $\begin{array}{l}33.94 \\
(5.82)\end{array}$ & 39.43 & 38.67 & 39.05 & 20.22 & 20.67 & 20.45 & 16.59 & 18.01 & 17.33 \\
\hline $\mathbf{T}_{8}$ & Pendimethalin (PE)-1.0 kg ai/ha & $\begin{array}{l}36.54 \\
(6.04)\end{array}$ & $\begin{array}{l}37.53 \\
(6.13)\end{array}$ & $\begin{array}{l}37.04 \\
(6.09)\end{array}$ & 33.88 & 33.26 & 33.57 & 22.79 & 23.19 & 22.99 & 17.27 & 18.46 & 17.88 \\
\hline $\mathbf{T}_{9}$ & Pendimethalin (PE)-1.5 kg ai/ha+ $1 \mathrm{HW}$ at $45 \mathrm{DAT}$ & $\begin{array}{l}28.43 \\
(5.33)\end{array}$ & $\begin{array}{l}29.44 \\
(5.43)\end{array}$ & $\begin{array}{l}28.93 \\
(5.38)\end{array}$ & 48.50 & 47.59 & 48.04 & 16.85 & 17.29 & 17.08 & 15.73 & 17.00 & 16.38 \\
\hline $\mathbf{T}_{10}$ & $\begin{array}{l}\text { Pendimethalin }(\mathrm{PE})-1.5 \mathrm{~kg} \text { ai/ } / \mathrm{ha}+2 \mathrm{HW} \text { at } 45 \text { and } 60 \\
\text { DAT }\end{array}$ & $\begin{array}{l}4.25 \\
(2.06)\end{array}$ & $\begin{array}{l}5.25 \\
(2.29)\end{array}$ & $\begin{array}{c}4.75 \\
(2.18)\end{array}$ & 92.33 & 90.68 & 91.50 & 12.61 & 13.17 & 12.89 & 5.52 & 7.47 & 6.53 \\
\hline $\mathbf{T}_{11}$ & Pendimethalin (PE)-1.0 kg ai/ha + $1 \mathrm{HW}$ at $45 \mathrm{DAT}$ & $\begin{array}{l}28.66 \\
(5.35)\end{array}$ & $\begin{array}{l}29.65 \\
(5.44)\end{array}$ & $\begin{array}{l}29.16 \\
(5.40)\end{array}$ & 48.16 & 47.29 & 47.72 & 18.26 & 18.74 & 18.50 & 15.96 & 17.43 & 16.72 \\
\hline $\mathbf{T}_{12}$ & $\begin{array}{l}\text { Pendimethalin (PE)-1.0 kg ai } / \mathrm{ha}+2 \mathrm{HW} \text { at } 45 \text { and } 60 \\
\text { DAT }\end{array}$ & $\begin{array}{l}14.08 \\
(3.75)\end{array}$ & $\begin{array}{l}15.07 \\
(3.88)\end{array}$ & $\begin{array}{l}14.57 \\
(3.82)\end{array}$ & 74.49 & 73.17 & 73.82 & 14.59 & 15.14 & 14.87 & 9.85 & 11.53 & 10.72 \\
\hline $\mathbf{T}_{13}$ & Weed free check & $\begin{array}{c}0.00 \\
(0.71)\end{array}$ & $\begin{array}{c}0.00 \\
(0.71)\end{array}$ & $\begin{array}{c}0.00 \\
(0.71)\end{array}$ & $\begin{array}{c}100.0 \\
0\end{array}$ & $\begin{array}{c}100.0 \\
0\end{array}$ & $\begin{array}{c}100.0 \\
0\end{array}$ & 0.00 & 0.00 & 0.00 & 0.00 & 0.00 & 0.00 \\
\hline $\mathbf{T}_{14}$ & Unweeded check & $\begin{array}{l}55.27 \\
(7.43)\end{array}$ & $\begin{array}{l}56.24 \\
(7.50)\end{array}$ & $\begin{array}{l}55.76 \\
(7.47)\end{array}$ & 0.00 & 0.00 & 0.00 & 62.64 & 63.59 & 63.12 & 56.63 & 56.31 & 58.66 \\
\hline & Mean & $\begin{array}{l}44.14 \\
(6.24)\end{array}$ & $\begin{array}{l}23.40 \\
(4.48)\end{array}$ & $\begin{array}{l}24.32 \\
(4.59)\end{array}$ & $\begin{array}{l}23.86 \\
(4.54)\end{array}$ & 57.65 & 56.73 & 57.19 & 18.88 & 19.36 & 15.12 & 16.39 & 16.39 \\
\hline & S. Em \pm & 0.08 & 0.10 & 0.10 & 0.10 & 1.79 & 1.75 & 1.77 & 1.67 & 1.73 & 3.15 & 3.04 & 3.04 \\
\hline & C.D. @ 5\% & 0.24 & 0.33 & 0.32 & 0.32 & 5.62 & 5.44 & 5.53 & 5.10 & 5.37 & 9.42 & 9.05 & 9.05 \\
\hline
\end{tabular}


Table.3 Effect of weed control treatments on growth and yield parameters in chilli

\begin{tabular}{|c|c|c|c|c|c|c|c|c|c|c|}
\hline \multirow[t]{2}{*}{ Treatments } & \multirow[t]{2}{*}{ Treatment details } & \multicolumn{3}{|c|}{$\begin{array}{c}\text { Fruit weight per plant } \\
\text { (kg) }\end{array}$} & \multicolumn{3}{|c|}{ Yield per plot (kg) } & \multicolumn{3}{|c|}{ Yield per ha(q) } \\
\hline & & $\begin{array}{l}\text { I } \\
\text { year }\end{array}$ & $\begin{array}{l}\text { II } \\
\text { year }\end{array}$ & Pooled & $\begin{array}{l}\text { I } \\
\text { year }\end{array}$ & $\begin{array}{l}\text { II } \\
\text { year }\end{array}$ & Pooled & $\begin{array}{l}\text { I } \\
\text { year }\end{array}$ & $\begin{array}{l}\text { II } \\
\text { year }\end{array}$ & Pooled \\
\hline $\mathbf{T}_{1}$ & Alachlor (PE)-1.5 kg ai/ha & 0.94 & 0.95 & 0.95 & 35.80 & 36.18 & 35.99 & 221.00 & 223.35 & 222.18 \\
\hline $\mathbf{T}_{2}$ & Alachlor (PE)-1.0 kg ai/ha & 0.92 & 0.93 & 0.92 & 34.88 & 35.26 & 35.07 & 215.30 & 217.65 & 216.47 \\
\hline $\mathbf{T}_{3}$ & Alachlor (PE)-1.5 kg ai $/ \mathrm{ha}+1 \mathrm{HW}$ at $45 \mathrm{DAT}$ & 0.99 & 1.00 & 0.99 & 37.48 & 37.86 & 37.67 & 231.36 & 233.71 & 232.53 \\
\hline $\mathbf{T}_{\mathbf{4}}$ & Alachlor (PE)-1.5 kg ai/ha $+2 \mathrm{HW}$ at 45 and $60 \mathrm{DAT}$ & 1.08 & 1.09 & 1.08 & 40.86 & 41.24 & 41.05 & 252.24 & 254.59 & 253.41 \\
\hline $\mathbf{T}_{5}$ & Alachlor (PE)-1.0 kg ai $/ \mathrm{ha}+1 \mathrm{HW}$ at $45 \mathrm{DAT}$ & 0.98 & 0.99 & 0.98 & 37.32 & 37.70 & 37.51 & 230.38 & 232.73 & 231.55 \\
\hline $\mathbf{T}_{6}$ & Alachlor (PE)-1.0 kg ai/ha $+2 \mathrm{HW}$ at 45 and $60 \mathrm{DAT}$ & 1.01 & 1.02 & 1.02 & 38.41 & 38.79 & 38.60 & 237.10 & 239.45 & 238.27 \\
\hline $\mathbf{T}_{7}$ & Pendimethalin (PE)-1.5 kg ai/ha & 0.93 & 0.94 & 0.94 & 35.35 & 35.73 & 35.54 & 218.23 & 220.58 & 219.40 \\
\hline $\mathbf{T}_{8}$ & Pendimethalin (PE)-1.0 kg ai/ha & 0.90 & 0.91 & 0.91 & 34.20 & 34.58 & 34.39 & 211.11 & 213.46 & 212.28 \\
\hline $\mathbf{T}_{\mathbf{9}}$ & Pendimethalin (PE)-1.5 kg ai/ha+ $1 \mathrm{HW}$ at $45 \mathrm{DAT}$ & 0.97 & 0.98 & 0.97 & 36.79 & 37.17 & 36.98 & 227.09 & 229.43 & 228.26 \\
\hline $\mathbf{T}_{10}$ & $\begin{array}{l}\text { Pendimethalin }(\mathrm{PE})-1.5 \mathrm{~kg} \text { ai/ha }+2 \mathrm{HW} \text { at } 45 \text { and } 60 \\
\text { DAT }\end{array}$ & 1.03 & 1.04 & 1.04 & 39.16 & 39.54 & 39.35 & 241.70 & 244.05 & 242.87 \\
\hline $\mathbf{T}_{11}$ & Pendimethalin (PE)-1.0 kg ai/ha $+1 \mathrm{HW}$ at $45 \mathrm{DAT}$ & 0.95 & 0.96 & 0.96 & 36.23 & 36.61 & 36.42 & 223.63 & 225.97 & 224.80 \\
\hline $\mathbf{T}_{12}$ & $\begin{array}{l}\text { Pendimethalin (PE)-1.0 kg ai/ha }+2 \mathrm{HW} \text { at } 45 \text { and } 60 \\
\text { DAT }\end{array}$ & 1.00 & 1.01 & 1.00 & 37.87 & 38.25 & 38.06 & 233.76 & 236.11 & 234.93 \\
\hline $\mathbf{T}_{13}$ & Weed free check & 1.11 & 1.13 & 1.12 & 42.21 & 42.97 & 42.59 & 260.56 & 265.25 & 262.90 \\
\hline $\mathbf{T}_{14}$ & Unweeded check & 0.44 & 0.43 & 0.41 & 16.53 & 16.34 & 16.44 & 102.04 & 100.86 & 101.45 \\
\hline & Mean & 0.95 & 0.96 & 0.95 & 35.94 & 36.30 & 36.12 & 221.82 & 224.08 & 222.95 \\
\hline & S. Em \pm & 0.03 & 0.04 & 0.04 & 1.33 & 1.44 & 1.38 & 8.19 & 8.87 & 8.52 \\
\hline & C.D. @ 5\% & 0.09 & 0.12 & 0.15 & 3.65 & 4.32 & 4.18 & 24.90 & 27.10 & 26.29 \\
\hline
\end{tabular}


Table.4 Effect of weed control treatments on yield and yield attributes in chilli

\begin{tabular}{|c|c|c|c|c|c|c|c|c|c|c|}
\hline \multirow[t]{2}{*}{ Treatments } & \multirow[t]{2}{*}{ Treatment details } & \multicolumn{3}{|c|}{$\begin{array}{c}\text { Fruit weight per plant } \\
\qquad(\mathrm{kg})\end{array}$} & \multicolumn{3}{|c|}{ Yield per plot (kg) } & \multicolumn{3}{|c|}{ Yield per ha(q) } \\
\hline & & $\begin{array}{c}\text { I } \\
\text { year }\end{array}$ & $\begin{array}{c}\text { II } \\
\text { year }\end{array}$ & Pooled & $\begin{array}{c}\text { I } \\
\text { year }\end{array}$ & $\begin{array}{c}\text { II } \\
\text { year }\end{array}$ & Pooled & $\begin{array}{c}\text { I } \\
\text { year }\end{array}$ & $\begin{array}{c}\text { II } \\
\text { year }\end{array}$ & Pooled \\
\hline $\mathbf{T}_{1}$ & Alachlor (PE)-1.5 kg ai/ha & 0.94 & 0.95 & 0.95 & 35.80 & 36.18 & 35.99 & 221.00 & 223.35 & 222.18 \\
\hline $\mathbf{T}_{2}$ & Alachlor (PE)-1.0 kg ai/ha & 0.92 & 0.93 & 0.92 & 34.88 & 35.26 & 35.07 & 215.30 & 217.65 & 216.47 \\
\hline $\mathbf{T}_{3}$ & Alachlor (PE)-1.5 kg ai/ha $+1 \mathrm{HW}$ at $45 \mathrm{DAT}$ & 0.99 & 1.00 & 0.99 & 37.48 & 37.86 & 37.67 & 231.36 & 233.71 & 232.53 \\
\hline $\mathbf{T}_{4}$ & Alachlor (PE)-1.5 kg ai/ha $+2 \mathrm{HW}$ at 45 and $60 \mathrm{DAT}$ & 1.08 & 1.09 & 1.08 & 40.86 & 41.24 & 41.05 & 252.24 & 254.59 & 253.41 \\
\hline $\mathbf{T}_{5}$ & Alachlor (PE)-1.0 kg ai/ha + $1 \mathrm{HW}$ at $45 \mathrm{DAT}$ & 0.98 & 0.99 & 0.98 & 37.32 & 37.70 & 37.51 & 230.38 & 232.73 & 231.55 \\
\hline $\mathbf{T}_{6}$ & Alachlor (PE)-1.0 kg ai/ha $+2 \mathrm{HW}$ at 45 and $60 \mathrm{DAT}$ & 1.01 & 1.02 & 1.02 & 38.41 & 38.79 & 38.60 & 237.10 & 239.45 & 238.27 \\
\hline $\mathbf{T}_{7}$ & Pendimethalin (PE)-1.5 kg ai/ha & 0.93 & 0.94 & 0.94 & 35.35 & 35.73 & 35.54 & 218.23 & 220.58 & 219.40 \\
\hline $\mathbf{T}_{8}$ & Pendimethalin (PE)-1.0 kg ai/ha & 0.90 & 0.91 & 0.91 & 34.20 & 34.58 & 34.39 & 211.11 & 213.46 & 212.28 \\
\hline $\mathbf{T}_{9}$ & Pendimethalin (PE)-1.5 kg ai/ha+ $1 \mathrm{HW}$ at $45 \mathrm{DAT}$ & 0.97 & 0.98 & 0.97 & 36.79 & 37.17 & 36.98 & 227.09 & 229.43 & 228.26 \\
\hline $\mathbf{T}_{10}$ & $\begin{array}{l}\text { Pendimethalin }(\mathrm{PE})-1.5 \mathrm{~kg} \text { ai } / \mathrm{ha}+2 \mathrm{HW} \text { at } 45 \text { and } 60 \\
\text { DAT }\end{array}$ & 1.03 & 1.04 & 1.04 & 39.16 & 39.54 & 39.35 & 241.70 & 244.05 & 242.87 \\
\hline $\mathbf{T}_{11}$ & Pendimethalin (PE)-1.0 kg ai/ha $+1 \mathrm{HW}$ at $45 \mathrm{DAT}$ & 0.95 & 0.96 & 0.96 & 36.23 & 36.61 & 36.42 & 223.63 & 225.97 & 224.80 \\
\hline $\mathbf{T}_{12}$ & $\begin{array}{l}\text { Pendimethalin (PE)-1.0 kg ai/ha }+2 \mathrm{HW} \text { at } 45 \text { and } 60 \\
\text { DAT }\end{array}$ & 1.00 & 1.01 & 1.00 & 37.87 & 38.25 & 38.06 & 233.76 & 236.11 & 234.93 \\
\hline $\mathbf{T}_{13}$ & Weed free check & 1.11 & 1.13 & 1.12 & 42.21 & 42.97 & 42.59 & 260.56 & 265.25 & 262.90 \\
\hline $\mathbf{T}_{14}$ & Unweeded check & 0.44 & 0.43 & 0.41 & 16.53 & 16.34 & 16.44 & 102.04 & 100.86 & 101.45 \\
\hline & Mean & 0.95 & 0.96 & 0.95 & 35.94 & 36.30 & 36.12 & 221.82 & 224.08 & 222.95 \\
\hline & S. $\operatorname{Em} \pm$ & 0.03 & 0.04 & 0.04 & 1.33 & 1.44 & 1.38 & 8.19 & 8.87 & 8.52 \\
\hline & C.D. @ 5\% & 0.09 & 0.12 & 0.15 & 3.65 & 4.32 & 4.18 & 24.90 & 27.10 & 26.29 \\
\hline
\end{tabular}


Table.5 Effect of weed control treatments on growth parameters in onion

\begin{tabular}{|c|c|c|c|c|c|c|c|}
\hline \multirow[t]{2}{*}{ Treatments } & \multirow[t]{2}{*}{ Treatment details } & \multicolumn{3}{|c|}{$\begin{array}{l}\text { Plant height at } 90 \text { DAT } \\
(\mathrm{cm})\end{array}$} & \multicolumn{3}{|c|}{$\begin{array}{c}\text { No. of leaves per plant } \\
\text { at } 90 \text { DAT }\end{array}$} \\
\hline & & $\begin{array}{c}\text { I } \\
\text { year }\end{array}$ & $\begin{array}{c}\text { II } \\
\text { year }\end{array}$ & Pooled & $\begin{array}{c}\text { I } \\
\text { year }\end{array}$ & $\begin{array}{c}\text { II } \\
\text { year }\end{array}$ & Pooled \\
\hline $\mathbf{T}_{1}$ & Alachlor (PE)-1.5 kg ai/ha & 52.20 & 53.10 & 52.65 & 12.88 & 13.53 & 13.20 \\
\hline $\mathbf{T}_{2}$ & Alachlor (PE)-1.0 kg ai/ha & 50.70 & 51.60 & 51.15 & 12.43 & 13.18 & 12.80 \\
\hline $\mathbf{T}_{3}$ & Alachlor (PE)-1.5 kg ai/ha $+1 \mathrm{HW}$ at $45 \mathrm{DAT}$ & 56.00 & 56.80 & 56.40 & 13.78 & 14.33 & 14.05 \\
\hline $\mathbf{T}_{4}$ & Alachlor (PE)-1.5 kg ai/ha $+2 \mathrm{HW}$ at 45 and $60 \mathrm{DAT}$ & 59.20 & 60.00 & 59.60 & 14.98 & 15.60 & 15.29 \\
\hline $\mathbf{T}_{5}$ & Alachlor (PE)-1.0 kg ai/ha $+1 \mathrm{HW}$ at $45 \mathrm{DAT}$ & 55.40 & 56.30 & 55.85 & 13.53 & 14.28 & 13.90 \\
\hline $\mathbf{T}_{6}$ & Alachlor (PE)-1.0 kg ai/ha $+2 \mathrm{HW}$ at 45 and $60 \mathrm{DAT}$ & 57.00 & 57.95 & 57.47 & 14.48 & 15.08 & 14.78 \\
\hline $\mathbf{T}_{7}$ & Pendimethalin (PE)-1.5 kg ai/ha & 51.20 & 52.00 & 51.60 & 12.58 & 13.33 & 12.95 \\
\hline $\mathbf{T}_{8}$ & Pendimethalin (PE)-1.0 kg ai/ha & 50.50 & 51.40 & 50.95 & 12.08 & 12.83 & 12.45 \\
\hline $\mathbf{T}_{9}$ & Pendimethalin (PE)-1.5 kg ai/ha+ $1 \mathrm{HW}$ at $45 \mathrm{DAT}$ & 53.90 & 54.80 & 54.35 & 13.28 & 13.78 & 13.53 \\
\hline $\mathbf{T}_{10}$ & Pendimethalin $(\mathrm{PE})-1.5 \mathrm{~kg}$ ai $/ \mathrm{ha}+2 \mathrm{HW}$ at 45 and $60 \mathrm{DAT}$ & 57.90 & 58.70 & 58.30 & 14.58 & 15.33 & 14.95 \\
\hline $\mathbf{T}_{11}$ & Pendimethalin $(\mathrm{PE})-1.0 \mathrm{~kg}$ ai $/ \mathrm{ha}+1 \mathrm{HW}$ at $45 \mathrm{DAT}$ & 52.70 & 53.75 & 53.22 & 13.11 & 13.56 & 13.34 \\
\hline $\mathbf{T}_{12}$ & Pendimethalin (PE)-1.0 kg ai/ha $+2 \mathrm{HW}$ at 45 and $60 \mathrm{DAT}$ & 56.60 & 57.50 & 57.05 & 14.18 & 14.93 & 14.55 \\
\hline $\mathbf{T}_{13}$ & Weed free check & 59.80 & 60.90 & 60.35 & 15.43 & 16.33 & 15.88 \\
\hline $\mathbf{T}_{14}$ & Unweeded check & 48.80 & 49.55 & 49.17 & 11.88 & 12.33 & 12.10 \\
\hline & Mean & 54.42 & 55.31 & 54.86 & 13.51 & 14.17 & 13.84 \\
\hline & S. Em \pm & 2.12 & 2.13 & 2.12 & 0.55 & 0.52 & 0.53 \\
\hline & C.D. @ 5\% & 6.01 & 6.09 & 6.05 & 1.63 & 1.50 & 1.24 \\
\hline
\end{tabular}


Table.6 Effect of weed control treatments on yield and yield attributes in onion

\begin{tabular}{|c|c|c|c|c|c|c|c|c|c|c|c|c|c|}
\hline \multirow[t]{2}{*}{ Treatments } & \multirow[t]{2}{*}{ Treatment details } & \multicolumn{3}{|c|}{$\begin{array}{l}\text { Dry weight of plant at } \\
\text { 90 DAT (g) }\end{array}$} & \multicolumn{3}{|c|}{ Yield per plant $(g)$} & \multicolumn{3}{|c|}{ Yield per plot $(\mathrm{kg})$} & \multicolumn{3}{|c|}{ Yield per ha $(q)$} \\
\hline & & $\begin{array}{c}\text { I } \\
\text { year }\end{array}$ & $\begin{array}{c}\text { II } \\
\text { year }\end{array}$ & Pooled & $\begin{array}{c}\text { I } \\
\text { year }\end{array}$ & $\begin{array}{c}\text { II } \\
\text { year }\end{array}$ & Pooled & $\begin{array}{c}\text { I } \\
\text { year }\end{array}$ & $\begin{array}{c}\text { II } \\
\text { year }\end{array}$ & Pooled & $\begin{array}{c}\text { I } \\
\text { year }\end{array}$ & $\begin{array}{c}\text { II } \\
\text { year }\end{array}$ & Pooled \\
\hline $\mathbf{T}_{1}$ & Alachlor (PE)-1.5 kg ai/ha & 5.14 & 5.44 & 5.29 & 69.55 & 71.45 & 70.50 & 31.30 & 32.15 & 31.73 & 193.19 & 198.47 & 195.83 \\
\hline $\mathbf{T}_{2}$ & Alachlor (PE)-1.0 kg ai/ha & 4.71 & 5.01 & 4.86 & 69.07 & 70.77 & 69.92 & 31.08 & 31.85 & 31.46 & 191.86 & 196.58 & 194.22 \\
\hline $\mathbf{T}_{3}$ & Alachlor (PE)-1.5 kg ai/ha $+1 \mathrm{HW}$ at $45 \mathrm{DAT}$ & 6.14 & 6.44 & 6.29 & 72.16 & 74.11 & 73.14 & 32.47 & 33.35 & 32.91 & 200.44 & 205.86 & 203.15 \\
\hline $\mathbf{T}_{4}$ & Alachlor (PE)-1.5 kg ai/ha $+2 \mathrm{HW}$ at 45 and $60 \mathrm{DAT}$ & 7.37 & 7.77 & 7.57 & 78.72 & 80.59 & 79.66 & 35.42 & 36.27 & 35.84 & 218.67 & 223.86 & 221.26 \\
\hline $\mathbf{T}_{5}$ & Alachlor (PE)-1.0 kg ai/ha $+1 \mathrm{HW}$ at $45 \mathrm{DAT}$ & 5.82 & 5.97 & 5.89 & 70.48 & 72.48 & 71.48 & 31.72 & 32.62 & 32.17 & 195.78 & 201.33 & 198.56 \\
\hline $\mathbf{T}_{6}$ & Alachlor (PE)-1.0 kg ai/ha $+2 \mathrm{HW}$ at 45 and $60 \mathrm{DAT}$ & 6.70 & 6.90 & 6.80 & 76.58 & 78.51 & 77.55 & 34.46 & 35.33 & 34.90 & 212.72 & 218.08 & 215.40 \\
\hline $\mathbf{T}_{7}$ & Pendimethalin (PE)-1.5 kg ai/ha & 4.85 & 5.15 & 5.00 & 69.10 & 71.10 & 70.10 & 31.10 & 32.00 & 31.55 & 191.94 & 197.50 & 194.72 \\
\hline $\mathbf{T}_{8}$ & Pendimethalin (PE)-1.0 kg ai/ha & 4.53 & 4.83 & 4.68 & 68.67 & 70.67 & 69.67 & 30.90 & 31.80 & 31.35 & 190.75 & 196.31 & 193.53 \\
\hline $\mathbf{T}_{9}$ & Pendimethalin (PE)-1.5 kg ai/ha+ $1 \mathrm{HW}$ at $45 \mathrm{DAT}$ & 5.68 & 5.83 & 5.75 & 69.94 & 71.94 & 70.94 & 31.47 & 32.37 & 31.92 & 194.28 & 199.83 & 197.06 \\
\hline $\mathbf{T}_{10}$ & $\begin{array}{l}\text { Pendimethalin }(\mathrm{PE})-1.5 \mathrm{~kg} \text { ai } / \mathrm{ha}+2 \mathrm{HW} \text { at } 45 \text { and } 60 \\
\text { DAT }\end{array}$ & 7.14 & 7.44 & 7.29 & 78.34 & 80.22 & 79.28 & 35.25 & 36.10 & 35.68 & 217.61 & 222.83 & 220.22 \\
\hline $\mathbf{T}_{11}$ & Pendimethalin (PE)-1.0 kg ai/ha $+1 \mathrm{HW}$ at $45 \mathrm{DAT}$ & 5.35 & 5.65 & 5.50 & 69.65 & 71.60 & 70.63 & 31.34 & 32.22 & 31.78 & 193.47 & 198.89 & 196.18 \\
\hline $\mathbf{T}_{12}$ & $\begin{array}{l}\text { Pendimethalin (PE)-1.0 kg ai/ha }+2 \mathrm{HW} \text { at } 45 \text { and } 60 \\
\text { DAT }\end{array}$ & 6.42 & 6.72 & 6.57 & 74.71 & 76.71 & 75.71 & 33.62 & 34.52 & 34.07 & 207.53 & 213.08 & 210.31 \\
\hline $\mathbf{T}_{13}$ & Weed free check & 7.75 & 8.25 & 8.00 & 82.87 & 86.72 & 84.80 & 37.29 & 39.02 & 38.16 & 230.19 & 240.89 & 235.54 \\
\hline $\mathbf{T}_{14}$ & Unweeded check & 4.21 & 4.36 & 4.28 & 36.02 & 37.87 & 36.95 & 16.21 & 17.04 & 15.78 & 100.06 & 105.19 & 102.63 \\
\hline & Mean & 5.84 & 6.12 & 5.98 & 70.42 & 72.48 & 71.45 & 31.69 & 32.62 & 32.09 & 195.61 & 201.34 & 198.47 \\
\hline & S. Em \pm & 0.53 & 0.55 & 0.54 & 2.57 & 2.64 & 2.60 & 1.16 & 1.19 & 1.21 & 7.13 & 7.35 & 7.23 \\
\hline & C.D. @ 5\% & 1.58 & 1.64 & 1.60 & 7.94 & 7.87 & 7.78 & 3.92 & 3.57 & 3.66 & 20.39 & 21.30 & 20.79 \\
\hline
\end{tabular}


Table.7 Effect of weed control treatments on economics feasibility in chilli +onion intercropping

\begin{tabular}{|c|c|c|c|c|c|}
\hline $\begin{array}{l}\text { Treat } \\
\text { ments }\end{array}$ & Treatment details & $\begin{array}{c}\text { Gross } \\
\text { returns } \\
(\mathbf{R s})\end{array}$ & $\begin{array}{l}\text { Cost of } \\
\text { cultivati } \\
\text { on } \\
\text { (Rs) }\end{array}$ & $\begin{array}{l}\text { Net } \\
\text { return } \\
(\text { Rs })\end{array}$ & $\begin{array}{l}\text { B:C } \\
\text { ratio }\end{array}$ \\
\hline $\mathbf{T}_{1}$ & Alachlor (PE)-1.5 kg ai/ha & $2,67,754$ & 85,300 & $1,82,454$ & 3.13 \\
\hline $\mathbf{T}_{2}$ & Alachlor (PE)-1.0 kg ai/ha & $2,63,611$ & 85,000 & $1,78,611$ & 3.10 \\
\hline $\mathbf{T}_{3}$ & Alachlor (PE)-1.5 kg ai/ha $+1 \mathrm{HW}$ at $45 \mathrm{DAT}$ & $2,78,785$ & 87,300 & $1,91,485$ & 3.19 \\
\hline $\mathbf{T}_{4}$ & Alachlor (PE)-1.5 kg ai/ha $+2 \mathrm{HW}$ at 45 and $60 \mathrm{DAT}$ & $3,03,713$ & 89,300 & $2,14,413$ & 3.40 \\
\hline $\mathbf{T}_{5}$ & Alachlor (PE)-1.0 kg ai/ha $+1 \mathrm{HW}$ at $45 \mathrm{DAT}$ & $2,74,623$ & 87,000 & $1,87,323$ & 3.15 \\
\hline$T_{6}$ & Alachlor (PE)-1.0 kg ai/ha $+2 \mathrm{HW}$ at 45 and $60 \mathrm{DAT}$ & $2,91,455$ & 89,000 & $2,02,455$ & 3.27 \\
\hline $\mathbf{T}_{7}$ & Pendimethalin (PE)-1.5 kg ai/ha & $2,65,476$ & 85,075 & $1,80,401$ & 3.12 \\
\hline $\mathbf{T}_{8}$ & Pendimethalin (PE)-1.0 kg ai/ha & $2,60,964$ & 84,850 & $1,76,114$ & 3.07 \\
\hline $\mathbf{T}_{9}$ & Pendimethalin (PE)-1.5 kg ai/ha+ $1 \mathrm{HW}$ at $45 \mathrm{DAT}$ & $2,71,778$ & 87,075 & $1,84,703$ & 3.12 \\
\hline $\mathbf{T}_{10}$ & $\begin{array}{l}\text { Pendimethalin (PE)-1.5 kg ai/ha }+2 \mathrm{HW} \text { at } 45 \text { and } 60 \\
\text { DAT }\end{array}$ & $2,97,611$ & 89,075 & $2,08,536$ & 3.34 \\
\hline $\mathbf{T}_{11}$ & Pendimethalin (PE)-1.0 kg ai/ha $+1 \mathrm{HW}$ at $45 \mathrm{DAT}$ & $2,69,344$ & 86,850 & $1,82,494$ & 3.10 \\
\hline $\mathbf{T}_{12}$ & $\begin{array}{l}\text { Pendimethalin (PE)-1.0 kg ai/ha }+2 \mathrm{HW} \text { at } 45 \text { and } 60 \\
\text { DAT }\end{array}$ & $2,85,713$ & 88,850 & $1,96,863$ & 3.21 \\
\hline $\mathbf{T}_{13}$ & Weed free check & $3,19,882$ & 98,400 & $2,21,482$ & 3.25 \\
\hline $\mathbf{T}_{14}$ & Unweeded check & $1,32,829$ & 84,400 & 48,429 & 1.57 \\
\hline \multicolumn{2}{|r|}{ Mean } & - & - & - & - \\
\hline \multicolumn{2}{|r|}{ S. $\mathbf{E m \pm}$} & - & - & - & - \\
\hline \multicolumn{2}{|r|}{ C.D. @ 5\% } & - & - & - & - \\
\hline
\end{tabular}


The effect of weed control treatments on economics of chilli +onion intercropping indicated that weed free check recorded highest gross returns (Rs.3,19,882) followed by $\mathrm{T}_{4}$ (Rs.3,03,713).Cost of cultivation was highest in weed free check (Rs.98,400) followed by $T_{4}$ (Rs.89,300). The higher cost of cultivation is due to increased labour charges incured during hand weeding. Highest $\mathrm{B}: \mathrm{C}$ ratio (3.40) was achieved by $\mathrm{T}_{4}$ followed by $\mathrm{T}_{10}$ (3.34) (Table 7). The higher $\mathrm{B}: \mathrm{C}$ ratio in $\mathrm{T}_{4}$ is due to higher net returns and lower cost of cultivation in comparison with weed free check. The results are in line with the findings reported by Biradar (1999), Gandolkar et al., (2015) and Chattopadhyay et al., (2016).

In conclusion, the pre emergence application of $1.5 \mathrm{~kg}$ of alachlor along with 2 hand weeding at 45 and 60 days after transplanting was found satisfactory in suppressing as well as controlling the weeds and obtaining higher yield of green chilli and onion followed by pendimethalin (PE)@ $1.5 \mathrm{~kg} / \mathrm{ha}+2 \mathrm{HW}$ under chilli+onion intercropping system.

\section{References}

Anonymous, 2017, www.nhb.in page no. 18 \& 22 . Biradar, S., 1999, Integrated weed management in chilli under northern transitional tract of Karnataka. M.Sc. (Agri.) Thesis, Univ. Agric. Sci., Dharwad (India).

Chattopadhyay, N., Mahalanabish, S., Hore, J.K. and Maity, T.K., 2016, Effect of different herbicides on growth and yield of onion. $J$ Crop Weed, 12 (1): 112-115.

Chaudhari, D. D., Patel, V. J., Patel, H. K., Aakashmishra, Patel, B. D. and Parmar, D. J., 2017, Integrated control of complex weed flora in garlic. Res. on Crops 18 (4): 668-674.
Dharmatti, P. R., Revanappa and Yashwanthakumar, K. H., 2008, Chemical weed control in drill sown onion. J. Asian Hort., 5(1): 38-40.

Gandolkar, K., Halikatti, S.I., Hiremath, S.M. and Pattar, P.S., 2015, Effect of sequential application of herbicides on weed management in drill sown onion under rainfed condition. Res. Environ. Life Sci., 8 (1): $1-4$

Gill, G. S. and Vijayakumar, 1969, Weed index- a new method for reporting weed control trials. Indian J. Agron., 16: 96-98.

Gomez, K. A. and Gomez, A. A., 1984, Statistical procedures for agricultural research. $2^{\text {nd }}$ edition, Singapore, Johnwilley \& Sons.

Kalasare, R.S., Ramesh Babu, Arvindkumar, B.N., Panpatte, D.G. and Shitap, M.S., 2016, Effect of herbicides on weeds in chilli+onion+cotton intercropping system. Bioinfolet, 13 (1A):6-9

Muthusankaranarayanan, A., Ali, A. M. and Veerendran, V., 1997, Weed smothering effect of intercrops in chilli. Indian J. Weed Sci. 29 (3 \& 4): 133-137.

Ningappa, 2013, Sequential application of pre and post emergent herbicides for weed management in chilli + onion + cotton intercropping system. M.Sc. (Agri.) Thesis, Univ. of Agril. Sciences, Dharwad (India).

Rajkumara,S., 2009, Weed management in onion -chilli cotton relay intercropping in rainfed vertisols. Ph.D. Thesis, University of Agricultural Sciences, Dharwad (India).

Shil, S. and Adhikary, P., 2014, weed management in transplanted chilli. Indian $J$ Weed Sci., 46(3): 261-263

Urraiya, P. and Jha, M.K., 2018, Weed management studies in kharif onion growth attributes. $J \quad$ Pharmacognosy Phytochemistry, 7 (1):1469-1471.

\section{How to cite this article:}

Vilas D. Gasti and Snehasish Chakravorty. 2019. Chemical Weed Control in Chilli + Onion Intercropping System. Int.J.Curr.Microbiol.App.Sci. 8(01): 3111-3121. doi: https://doi.org/10.20546/ijcmas.2019.801.332 